\title{
SOBRE LAS FORTUNATAE INSULAE DE PLINIO EL VIEJO
}

\author{
Antonio Tejera Gaspar \\ Catedrático de Arqueología - Universidad de La Laguna \\ atejera@ull.es_antejera@gmail.com
}

\section{RESUMEN}

En este trabajo propongo una serie de cuestiones, relativas al texto de Plinio el Viejo sobre las Afortunadas, donde destaco su importancia como documento histórico acerca del Descubrimiento de Canarias en la Antigüedad, en donde figura el nombre de las siete islas mayores que forman parte de este Archipiélago atlántico. Y pongo de manifiesto, asimismo, que, a excepción de la referencia a la isla Ombrios, como estudiaron A. Díaz Tejera y A. García, vinculada con uno de los espacios míticos ubicados en este mar, el resto puede ser analizado con los mismos criterios con los que se estudia cualquier otra fuente documental.

Palabras Clave: Afortunadas, Descubrimiento, Islas Canarias, Ombrios.

\section{REGARDING THE FORTUNATAE INSULAE IN PLINY THE ELDER}

\section{ABSTRACT}

In this paper I propose a series of questions regarding Pliny the Elder's text on the Fortunate Islands, in which I highlight its importance as a historical document on the discovery of the Canary Islands in antiquity, where the names of the seven major islands, which form part of this Atlantic archipelago, appear. And I also show that, with the exception of the reference to the island of Ombrios, as studied by A. Díaz Tejera and A. García, linked to one of the mythical spaces located in this sea, the rest can be analysed with the same criteria used to study any other documentary source.

KeYwords: Fortunate Islands, Discovery, Canary Islands, Ombrios.

Uno de los temas que, sin duda, ha sido muy debatido sobre las islas Canarias en la Antigüedad es el de su Descubrimiento y, por extensión, todo lo que se halla vinculado al poblamiento humano, puesto que sin ese acontecimiento no se puede explicar este otro. Y aunque no es el lugar apropiado para plantear en profundidad un problema de esas características que, como el del poblamiento, ha sido tratado en época reciente por Alicia García García y el autor de este trabajo ${ }^{1}$, me ha parecido oportuno, sin embargo, hacer algunas pocas consideraciones sobre el primero de los temas reseñados. Y aunque se trata de una cuestión estudiada, de forma recurrente desde hace mucho tiempo, existe una fecha y un autor de referencia a partir 
de la que el enfoque de su análisis ha ido cambiando, de manera lenta, todo hay que decirlo, pero que nos ha permitido, no obstante, replantear el problema de modo muy diferente a como había sido tratado en la historiografía canaria en las centurias precedentes. Este autor no es otro que el profesor Juan Álvarez Delgado y el trabajo que marca ese hito imprescindible es el de las «Islas Canarias en la Antigüedad», que lo dio a conocer el año 1945, en la Revista de Historia Canaria ${ }^{2}$. Y a partir de ahí, proliferaron una serie de investigadores y de estudios que nos han permitido entender el problema con una perspectiva bien distinta a como había sido concebido hasta esas fechas. En ese sentido, los variados análisis filológicos e históricos, e incluso los de carácter arqueológico, aunque en este aspecto solo hayan sido de manera tangencial, han contribuido, sin duda, a partir de este estudio pionero, a entenderlo de manera muy distinta. En todo caso, y para comprender debidamente su contenido, me parece necesario no perder de vista ninguna de las propuestas con las que ha sido analizado el texto pliniano del que en estas pocas líneas pretendo hacer unas cuantas reflexiones relativas a las Islas Afortunadas (Fortunatae Insulae), que se halla recogido en los parágrafos 202-205 del libro VI de la Historia Natural (Naturalis Historia) de Plinio el Viejo (Caius Plinius Secundus), escrita en el último tercio del siglo I de la Era, en época del emperador Vespasiano ${ }^{3}$. Y siguiendo, pues, las sugerencias que en su día expresara Álvarez Delgado, como veremos a continuación, conviene observar de antemano la precisión geográfica, e incluso descriptiva, con la que en el denominado texto de Plinio-Juba, como a él gustaba referirse, se ubica a las AfortunadasCanarias, así como la distancia en millas desde los puntos conocidos de Cádiz o Mogador, pero también el modo de navegar hacia ellas, así como los datos bien determinados para poder avistarlas. Pienso, pues, que los parágrafos de Plinio, a pesar de su escasa extensión, y que para algunos no es más que un texto confuso, como así ha sido considerado en la mayoría de las ocasiones, podremos comprobar que, a excepción de las líneas, ciertamente discutidas, dedicadas a la isla Ombrios ${ }^{4}$, como veremos, el resto del contenido no me parece que plantee ningún problema para considerarlo un documento de gran valor histórico, bien alejado, por cierto, de la opinión

${ }^{1}$ A. García García y A. Tejera Gaspar (2018): Bereberes contra Roma. Insurrecciones indígenas en el norte de África y el poblamiento de las Islas Canarias, LeCanarien Ediciones, Santa Cruz de Tenerife.

2 Álvarez Delgado, 1945: 26-61. Véanse, asimismo, los trabajos de Delgado Delgado, 20112012: 9-23; Díaz Tejera, 1988: 13-32; García García, 2008: 141-164; García García y Tejera Gaspar, 2014: 157-167; García-Talavera Casañas, 2008: 60-68; Jiménez González, 2014; Mederos Martín y Escribano Cobo, 2014; Santana Santana, Arcos Pereira, Atoche Peña y Martín Culebras, 2002: 230.

${ }^{3}$ Algunas de las ideas que recojo en este artículo tienen como precedente un texto mío, publicado en línea, en la revista BIEREHITE (2019, 2: 23-33), del Museo de la Historia de Tenerife (MHA), titulado «Un viaje a las Fortunatae Insulae» [http://doi.org/10.31939/bierehite/2019.02].

${ }^{4}$ En muchas ocasiones el nombre de esta isla aparece como Ombrion, y así figura en el texto. Este hecho ha producido una confusión con la denominación correcta de Ombrios, ya que la otra versión corresponde al mismo sustantivo, pero en acusativo. 
de los estudiosos que lo han rechazado por creerlo propio de la llamada literatura paradoxográfica, en la que se mezclan referencias geográficas más o menos precisas, entreveradas de alusiones a islas míticas o legendarias, antes que de una geografía real, que hoy, sin embargo, pueden ser bien contrastadas con criterios históricos y filológicos.

Una cuestión previa con relación al texto de Plinio, es que para su debida comprensión, su análisis no debe circunscribirse solo a los parágrafos 202-205, donde se contiene el núcleo esencial sobre las islas Afortunadas, sino que deben incluirse, también, los que le preceden, porque en ellos se encuentran diversas alusiones a islas que, en este caso y con buen criterio, han sido consideradas islas míticas o fabulosas. Sin tener en cuenta ese aspecto, no es posible, a mi juicio, entender el porqué de la existencia en estos párrafos del porqué de la presencia de la isla Ombrios -la Pluvialia de Estacio Seboso-, cuestión que ha sido bien estudiada en los trabajos de Alberto Díaz Tejera y Alicia García, como un ejemplo característico de lo que se conoce como islas míticas que pertenecen, en este caso sí, a un buen número de las que conformaron el imaginario grecolatino.

Como decía, para entender mi propuesta, me parece imprescindible hacer una primera consideración sobre el problema de la isla Ombrios-Pluvialia, para lo que seguiré el estudio realizado por los citados investigadores, al considerar que a Ombrios la creían una isla mítica, antes que un espacio real. Se fundamentaban para ello en la expresión que se recoge en el texto sobre la existencia de unos árboles "de los que se extrae agua, la de los árboles negros es amarga y la de los más claros, agradable de beber». En un estudio minucioso sobre esta cuestión, Díaz Tejera (1988: 31) compara el texto con otros, igualmente de fuerte raigambre mítica o poética, como las dos fuentes de las que habla Homero en la Odisea, o de los ríos de la Atlántida, donde, en su ribera, crecen «dos árboles extraños, de forma que quien coma las frutas de los del río de las penas se deshace en lágrimas, pero el que, por el contrario, coma las frutas de los del río de las alegrías no muere sino que rejuvenece» (Díaz Tejera, 1988: 31), quizá el documento que define mejor este carácter mítico del texto de Plinio es, sin duda, el de Pomponio Mela, referido, además, a las islas Afortunadas -aunque no se trate en este caso de las islas Canarias-, donde se distinguen, sobre todo, «...por la extraña naturaleza de dos fuentes: los que gusten de una de ellas se mueren de risa y el remedio para los que así son afectados consiste en beber de la otra» (Díaz Tejera, 1988: 31). Esta tradición mítico-poética que de modo similar se halla presente en el texto de Plinio, relativo a la citada isla, nos parece un buen argumento para desecharla, de manera evidente, como propia de una geografía real, demostrando de ese modo que nada tiene que ver con la tradicional vinculación que se ha hecho, de manera reiterada, con algunas otras, pero sobre todo con la de El Hierro, según figura en muchísimos cronistas y escritores. En el estudio de Alicia García García (2011), continuando la propuesta del profesor Díaz Tejera, se analiza, asimismo, y de forma minuciosa, el carácter mítico del término Ombrios-la lluvia-, teniendo presente que a Zeus se le atribuye, entre otros, este epíteto, uno más de los fenómenos característicos de la naturaleza a los que siempre se asimila esta divinidad, como el de Dios tonante -lanzador de truenos y rayos-, como suele ser representado, de manera frecuente, en distintas iconografías de materiales griegos, sobre todo en cerámicas. 
Por esa razón, no es de extrañar que los estudiosos del texto lo hubieran considerado en conjunto sospechoso de su veracidad, creyendo, del mismo modo, que la totalidad de lo que se contiene en él no sería otra cosa que una mera continuidad de lo que se halla en los parágrafos inmediatamente anteriores que pertenecen, como digo, a islas míticas o legendarias que, de ningún modo se pueden vincular, por el contrario, con los otros datos que, a mi juicio, son informaciones muy precisas sobre las Fortunatae Insulae. Creo que, exceptuando lo dicho, lo contenido en los otros párrafos resulta muy coherente desde la primera hasta la última línea, como trataré de expresarlo, aunque sin entrar en un estudio pormenorizado sobre las denominaciones de las islas y otras cuestiones que, por cierto, han sido ya bien estudiados, por lo que no precisa un análisis in extenso que no era mi intención tratarlo, ni es posible hacerlo en una publicación de estas características ${ }^{5}$, de modo que, a excepción de lo referido a la isla Ombrios, el texto es un documento excepcional que resulta totalmente coherente, por lo que puede ser considerado, en efecto, no solo la carta de nacimiento de las islas Canarias -así lo defendió Celso Martín de Guzmán-, sino también la primera imagen de este Archipiélago, como fue conocido durante toda la Antigüedad hasta que tuvo lugar su Redescubrimiento en el Medievo.

De las cuestiones de interés que me han parecido pertinentes destacar, la primera es, sin duda, las fuentes de conocimiento que fueron utilizadas por Plinio para componer los parágrafos 202-205 del citado libro VI de su Historia Natural. Se ha dicho, ciertamente, que nuestro autor es un compilador de informaciones de diversa procedencia, a partir de las que redactó su obra, y por lo que, debido a su propia manera de trabajar a base de fichas extractadas de quienes fue un curioso lector, se le ha criticado buena parte de su obra. Una apreciación que me resulta de poca entidad, desde luego, porque lo importante no es el modo en el que preparó su magna obra, sino lo que dice en ella y cómo lo dice. Y respecto a nuestras islas, ya nos advierte del origen variado de sus datos, porque, aunque parte sean, en efecto, anónimos, debió de recibirlos, asimismo, de informes de diversa índole, por lo que se explica la expresión vaga, cuando dice: Qui sunt...: «Hay quienes piensan que más allá de éstas [las islas Purpurarias] se encuentran las Afortunadas y algunas otras... $\nu^{6}$. ¿Quién o quiénes se esconden ante expresión tan poco definida? No resulta fácil saberlo, es cierto, pero es de suponer que esta información procedería de algunos marinos que, con anterioridad a lo aportado por Estacio Seboso y Juba, debieron de haber realizado distintas exploraciones por la costa africana, haciendo suponer que fueran ellos los que, primeramente, darían cuenta de su existencia, aunque nada sabemos con certeza de quién se trataba, ni cómo obtendría ese conocimiento. Entre los muchos nombres que se han propuesto se encuentra el del siempre socorrido Eudoxo de Cízico,

${ }^{5}$ Para los aspectos relacionados con la nesonimia de las islas, así como la perenne confusión entre islas reales e islas míticas puede verse la obra del profesor Marcos Martínez Hernández, 1992, 1996 y 2002.

${ }^{6}$ Las referencias al texto de Plinio proceden de la obra de Virgilio Bejarano (1987: 135). 
geógrafo y navegante de origen griego que vivió entre el año 150 a.C. y el 100 a.C., a quien se acude de forma reiterada para entrever lo dicho en un periplo que lleva su nombre, cuando al costear las riberas atlánticas africanas, quizá alcanzaría a ver los islotes que forman el Archipiélago Chinijo, al mismo tiempo que la isla de Lanzarote y, por extensión, Fuerteventura. Es posible, en efecto, que otros exploradores que siguieron esa misma ruta, con el fin de saber qué habría más allá de la isla de Kerne (Mogador) (Kbiri Alaoui y López Pardo, 1998; Marzoli y El Khayari, 2018), se hubieran encontrado con esos espacios geográficos, como así sucedería de manera reiterada a lo largo de la historia de la exploración del Océano Atlántico que bordea este continente. La segunda fuente es, sin duda, Estacio Seboso, tal como Plinio alude a él con su nombre, Seboso, y cita algunas islas que conoció: Junonia, Pluvialia, Capraria, Invallis y Planasia. Y, por último, el propio Juba que es, sin duda, la fuente principal.

La otra cuestión a la que me gustaría referirme he de hacerla, asimismo, en forma de pregunta y es la de saber ¿quién fue el descubridor de las islas?, y sobre todo, ¿cuándo tuvo lugar este acontecimiento? La respuesta no es otra que, de nuevo, un interrogante, ya que si nos ajustamos a lo dicho más arriba, en el análisis del texto solo podemos movernos en el terreno de la duda. Y si aceptáramos que, al menos las dos islas más orientales y, cercanas al continente africano, que figuran con la denominación específica de las Afortunadas, con los nombres de Invallis y Planasia, lo más que podríamos alcanzar a decir es que pudieron haber sido descubiertas, quizá a finales del siglo II a.C., aunque esto no pasa de ser más que una mera suposición.

Sin embargo, frente a lo que se ha generalizado en la historiografía sobre el descubrimiento de las islas Canarias, de que este hecho tuvo lugar en tiempos de Juba II de Mauritania, conviene decir, siguiendo siempre lo que se halla consignado en Plinio, que el primer descubridor de este Archipiélago podría haber sido, en efecto, Estacio Seboso, personaje de escaso perfil biográfico, pero del que sabemos fue «un poco anterior a Juba, viajero y amigo de Catulo, por lo que pudo reunir sus conocimientos en una extensa obra, que se conoce por medio de diversas referencias, como la que nos fue aportada conocida por Plinio", y que debió de haber vivido en torno al año 35 a.C. (Díaz Tejera, 1988: 14; Delgado Delgado, 2011-2012), por lo que la fecha a manejar se fijaría entonces hacia la mitad del siglo I a.C. Y, por tanto, su verdadero descubridor, aunque en este caso sí, de nombre conocido, podría ser el citado explorador latino, acontecimiento que, en ese caso, nos ayudaría a explicar un texto, en apariencia confuso, del cronista-historiador L. Torriani, quien, aunque nos precisa que este acontecimiento había tenido lugar durante el mandato del rey Juba II de Mauritania (25 a.C.-23 d.C.), añade un dato de especial interés cuando nos dice que «Algunos pretenden que estas islas quedaron después desiertas y casi desconocidas, durante muchos años, y que más tarde las volvió a descubrir Juba y las pobló con númidas» ${ }^{7}$, añadiendo que, en realidad se trataría de un Redescubrimiento, ya que

${ }^{7}$ Torriani, 1592 [1978]: 20, capítulo IV, «Quiénes fueron los primeros habitantes de estas islas». 
había sido conocido con anterioridad a la exploración del Archipiélago por mandato del rey mauritano. En ese caso, además del verdadero Redescubrimiento que tuvo lugar en el Medievo, en un espacio temporal que media entre los años 1291 y quizá 1312, desde la misma Antigüedad ya se podría hablar de un Redescubrimiento, aunque no sería tal, sino acaso un conocimiento mejor documentado de las islas por parte de Juba, ya que a la vista de su descripción, es evidente que el rey mauritano debió de saber todo lo que se decía de ellas y, desde luego, parece seguro que habría tenido conocimiento de los informes elaborados por Estacio Seboso sobre su descubrimiento. De este modo, sería posible concluir, con relativa seguridad, que el primer descubridor de este Archipiélago sería el citado explorador y su conocimiento podría fijarse, entonces, mediada la última centuria antes de la Era.

Otro de los problemas que ha sido objeto de mucha discusión -hasta el extremo de considerar que se trata de una confusión de Plinio o de una mala interpretación de lo contenido en los manuscritos que manejó- es la referencia que figura en el texto, cuando se habla de la posición de las Afortunadas, a las que sitúa al oeste de la costa atlántica africana, y sobre las que, además, nos aporta la distancia precisa y su concreta ubicación, bien diferente a la ruta occidental desde la que Plinio las describe. Sin embargo, un buen número de estudiosos del texto la han considerado de difícil aceptación cuando se dice que desde las islas, Pluvialia y Capraria, ubicadas en el Ocaso, es decir, hacia Occidente, hasta alcanzar las Afortunadas, situadas en el Orto, hay una distancia de «...250 millas (y) se encuentran frente a la costa izquierda de Mauritania y hacia el suroeste y que una se llama Invallis y la otra Planasia por su aspecto" (Bejarano, 1987: 135). Las 250 millas equivalen a unos $375 \mathrm{~km}$ que sería la distancia aproximada que existe desde La Palma hasta Lanzarote y Fuerteventura, de manera que tanto aquella como la ubicación al suroeste de la costa izquierda del litoral africano, no parecen dejar dudas de que se trata de las dos islas orientales, a las que singulariza con los nombres citados ${ }^{8}$. Y es aquí donde se producen, en apariencia, las confusiones del texto que han resultado muy difíciles de aceptar. Sin embargo, desde mi punto de vista esta referencia contiene una información de extraordinario interés. Y explicaré por qué.

Para entender lo que Plinio quiere expresar conviene volver al inicio del texto, cuando el naturalista habla de las islas Afortunadas y algunas otras, que luego las citará siguiendo lo descrito en los manuscritos de Estacio Seboso y de Juba II. La referencia aquí del término de las Afortunadas, Invallis y Planasia, me parece que antes de considerarlas una posible confusión de Plinio, la alusión a esas Afortunadas es,

${ }^{8}$ El profesor Díaz Tejera sugiere (1988: 21) que el nombre Invalis pudiera ser Nivalis, debido a una posible corrupción del manuscrito, en el que la letra I podría confundirse con una N, como es muy común en los inicios de palabra, por lo que pudiera decir Nivalis, que relaciona con Tenerife, pero el hecho de que las Afortunadas están cerca de África, los nombres de Nivalis y Planasia, serían en ese caso Lanzarote y Fuerteventura. 
como digo, un dato de gran relevancia porque se está refiriendo a un hecho que tiene una gran trascendencia en el conocimiento que los romanos debieron de haber ido adquiriendo de la costa africana después del año 146 a.C., a poco de haber conquistado y ocupado la ciudad púnica de Cartago, fecha a partir de la cual iniciarín la exploración de las zonas que, con anterioridad, habían sido las áreas de influencia de la civilización fenicio-púnica que había alcanzado, con seguridad, hasta los islotes de Mogador (Essauira). A partir de este punto y, en años posteriores, se iniciaría una exploración en la que participó, también, el historiador griego Polibio, muy amigo de Publio Cornelio Escipión el Africano, el conquistador de la ciudad de Cartago, de modo que este otro hecho debió de ocurrir entre el 145-144 a.C., a pesar de que desconocemos, además de la datación precisa, hasta qué latitud del Atlántico sur podrían haber llegado, pero no creemos, en ningún caso, que alcanzara alguna de las islas del Archipiélago. Hazaña que muchos estudiosos se la atribuyen, como he señalado, a Eudoxo de Cízico, del mismo modo que así lo defendería, entre otros, el profesor Álvarez Delgado. No obstante, en esta ocasión solo me interesa poner de manifiesto que el hallazgo de unas islas que, por extensión, se seguirían llamando aún Afortunadas, mantuvieron siempre el nombre clásico que durante siglos se les había atribuido a estas islas en el imaginario de griegos y latinos, de manera que las referidas de Seboso y Juba formarían parte de esas «algunas otras» que se descubrirían con posterioridad.

La comprensión, pues, de este fragmento resulta confuso, en apariencia, puesto que si está hablando de las islas Afortunadas por qué razón, después de hacer la descripción de algunas de ellas, alude en este punto a su existencia en las cercanías del continente. La primera impresión es que se trata, como decía, de un posible error, o acaso de una interpolación que resultaría contradictoria con el contenido general de la narración. No obstante, si estuviéramos en lo cierto, de que antes incluso del periplo de Estacio Seboso, las primeras islas descubiertas serían las orientales, y por este orden, Lanzarote y Fuerteventura, no resultaría extraño pensar que esas serían consideradas las Afortunadas, como así se denominarían, primero las del entorno de Cádiz y más tarde las de Mogador, hasta tanto se descubrieran estas dos, pertenecientes ahora al archipiélago canario, como era de esperar que así sucediera, a medida que se fueron descubriendo las que, en origen habían formado parte de unas islas míticas que desde hacía siglos se ubicaban hacia el extremo occidente por donde el sol que portaba las almas de los bienaventurados o afortunados cada día era engullido por el mar. En este contexto, la referencia a las Afortunadas, creo que, no solo no habría de ser considerado un error, sino que, por el contrario, enriquece el contenido de la información al confirmar ahora una realidad geográfica tangible, que durante siglos había sido parte esencial de su imaginario.

Para complementar la comprensión del texto, conviene tener en cuenta que el primer descubrimiento debió de hacerse siguiendo una ruta que se haría ciñéndose a la costa africana, lo que favorecería el descubrimiento de esas dos Afortunadas y, con posterioridad seguirían otra ruta, que se haría desde las occidentales acercándose hacia el continente, teniendo siempre presente las otras islas, aunque como digo, eso necesitaría un análisis muy detallado que no es posible realizar en esta ocasión. La ruta seguida sería primeramente por la costa africana y luego de Occidente a Oriente, 
como he dicho. Y si fuera correcta nuestra lectura aparecen de este modo las siete islas, siempre que se deseche, por obvio, la citada Ombrios. Esto es, Junonia Maior (¿La Palma?), [Junonia Minor (¿La Gomera?)], Capraria (¿El Hierro?), Ninguaria (¿Tenerife?), Canaria (¿Gran Canaria?), Invallis (¿Lanzarote?) y Planasia (¿Fuerteventura?).

Quisiera hacer, por último, una breve consideración acerca de otra de las cuestiones sobre las que se ha discutido mucho. Me refiero a las aludidas construcciones que se habían encontrado en algunas de las islas, aunque no tengamos de ella una ubicación determinada. Se ha discutido mucho sobre la referencia que se hace en el texto de Plinio-Juba, cuando en los últimos párrafos se habla de la existencia de "vestigios de edificaciones», lo que ha dado pie a distintas opiniones al respecto, como la de considerar que la isla podría ser Gran Canaria, y que con anterioridad podría haber sido, siquiera fuera de manera esporádica, ocupada desde época feniciopúnica, como así ha sido defendido por muchos investigadores, como se recoge en el libro de A. Santana, T. Arcos, P. Atoche y J. Martín (2002: 310). Y aunque solo sea en el ámbito de la especulación, conviene tener en cuenta que antes de que las islas figuraran en la Historia Natural de Plinio el Viejo, se realizarían una serie de navegaciones a las que nos hemos referido, como la del griego Eudoxo de Cízico y el romano Estacio Seboso, y quizá, antes de él, la de otros muchos expedicionarios de los que nada conocemos. En este contexto, pues, no sería extraño que existieran esos edificios a los que se refiere el texto, sino todo lo contrario, ya que muy bien podrían haber sido testimonios de esos viajeros o expedicionarios que, a lo largo del siglo I a.C. visitarían las islas, como he tratado de defender en este breve trabajo.

El texto, como es bien sabido, contiene otros muchos aspectos bien estudiados que han sido objeto, asimismo, de análisis certeros por quienes los han realizado, y que espero poder tratarlos, en otra ocasión, de manera más detenida.

\section{REFERENCIAS BIBLIOGRÁFICAS}

Álvarez Delgado, J. (1945): «Las islas Afortunadas en Plinio», Revista de Historia [Canaria] 69: 26-61.

Álvarez Delgado, J. (2014): Descubrimiento, colonización y primer poblamiento de las Islas Canarias, edición de Alfredo Mederos Martín y Gabriel Escribano Cobo, Ediciones Idea, Col. Thesaurus, Santa Cruz de Tenerife.

Bejarano, V. (1987): «Hispania Antigua en la Historia Naturab, en Fontes Hispaniae Antiquae (FHA), fasc. VII, Institut d'Arquelogia i Prehistòria, Barcelona.

Delgado Delgado, J. A. (2011-2012): «Canarias en la antigüedad como problema histórico», Tabona: Revista de Prehistoria y de Arqueología 19: 9-23.

Díaz Tejera, A. (1988): «Las Canarias en la Antigüedad», en Francisco Morales Padrón (ed.), Gran Enciclopedia de España y América. Canarias y América, Espasa-Calpe - Argantonio, Madrid, pp. 13-32.

García GarCía, A. (2008): «El informe de Juba II sobre las Fortunatae Insulae (Plinio el Viejo, $H N$, VI, 202-205)", Tabona: Revista de Prehistoria y de Arqueólogía 17: 141-164.

García García, A. (2011): «Ombrios, Isla de la lluvia, en las Fortunatae Insulae de Plinio el Viejo», en Marcos Martínez Hernández, Fremiot Hernández González, Luis Miguel Pino Campos 
(coords.), Sodalium Munera. Homenaje a Francisco González Luis. Ediciones Clásicas, Madrid, pp. 167-176.

García García, A. - Tejera Gaspar, A. (2014): «La primera imagen de las Islas Canarias en la Naturalis Historia de Plinio el Viejo", Fortunatae 25: 157-167.

García-TAlaVera Casañas, F. (2006): «Purpurarias afortunadas: la Macaronesia Central en la Antigüedad", Makaronesia: Boletín de la Asociación de Amigos del Museo de Ciencias Naturales de Tenerife 8: 60-82.

Jiménez GonZÁlez, J. J. (2014): La tribu de los CANARII. Arqueología, Antigüedad y Renacimiento, Le Canarien ediciones, Santa Cruz de Tenerife.

Kbiri Alaoui, M. - López Pardo, F. (1998): «La factoría fenicia de Mogador (Essauira, Marruecos): Las cerámicas pintadas», Archivo Español de Arqueología 71: 5-25.

Martínez Hernández, M. (1992): Canarias en la mitología. Historia mítica del Archipiélago, Centro de la Cultura Popular Canaria, Santa Cruz de Tenerife.

Martínez Hernández, M. (1996): Las Islas Canarias de la Antigüedad al Renacimiento. Nuevos aspectos, Centro de la Cultura Popular Canaria, Santa Cruz de Tenerife.

Martínez Hernández, M. (2002): Las Islas Canarias en la Antigüedad Clásica. Mito, historia e imaginario. Centro de la Cultura Popular Canaria, Santa Cruz de Tenerife.

Marzoli, D. - El KhaYARI, A. (2018): «Mogador (Essaouira), Marokko. Ein phönizischer Außenposten an der marokkanischen Atlantikküste. Die Arbeiten der Jahre bis 2018", iDAI.publications. Elektronische Publikationen des Deutschen Archäologischen Instituts 1: 72-75.

Mederos Martín, A. - Escribano Cobo, G. (eds.) (2014): Descubrimiento, colonización y primer poblamiento de las Islas Canarias [de Juan Álvarez Delgado], Ediciones Idea, Col. Thesaurus, Santa Cruz de Tenerife.

Santana Santana, A. - Arcos Pereira, T. - Atoche Peña, P. - Martín Culebras, J. (2002): El conocimiento geográfico de la costa noroccidental de África en Plinio: la posición de las Canarias, Olms Verlag, Hildesheim, Zürich, New York.

Tejera GasPar, A. (2019): «Un viaje a las Fortunatae Insulae», BIEREHITE 2: 23-33 [Museo de la Historia de Tenerife (MHA)] [http://doi.org/10.31939/bierehite/2019.02].

Torriani, L. (1592 [1978]): Descripción e Historia del Reino de las Islas Canarias antes Afortunadas, con el parecer de sus fortificaciones, traducción, introducción y notas de Alejandro CIORANESCU, Goya ediciones, Santa Cruz de Tenerife. 
